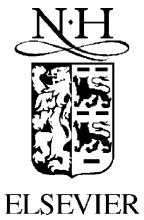

\title{
Fast randomized point location without preprocessing in two- and three-dimensional Delaunay triangulations
}

\author{
Ernst P. Mücke* ${ }^{*}$ Isaac Saias ${ }^{1}$, Binhai Zhu ${ }^{2}$ \\ Los Alamos National Laboratory, Los Alamos, NM, USA
}

Communicated by M.T. Goodrich; submitted 21 October 1996; accepted 2 February 1998

\begin{abstract}
This paper studies the point location problem in Delaunay triangulations without preprocessing and additional storage. The proposed procedure finds the query point by simply "walking through" the triangulation, after selecting a "good starting point" by random sampling. The analysis generalizes and extends a recent result for $d=2$ dimensions by proving this procedure takes expected time close to $\mathrm{O}\left(n^{1 /(d+1)}\right)$ for point location in Delaunay triangulations of $n$ random points in $d=3$ dimensions. Empirical results in both two and three dimensions show that this procedure is efficient in practice. ๑ 1999 Elsevier Science B.V. All rights reserved.
\end{abstract}

Keywords: Computational geometry; Geometric computing; Randomized algorithms; Delaunay triangulations; Point location; Three dimensional

\section{Introduction}

Point location is one of the classical problems in computational geometry and has various applications of practical relevance, for example, in the areas of geographic information systems (GIS) or computeraided design and engineering (CAD/CAE). The problem is well studied in the computational geometry literature and several theoretically optimal algorithms have been proposed; see for example, Snoeyink's recent survey [23]. Unfortunately, algorithms that are optimal in theory do not necessarily yield to good practical performance. This is also true in the case of point location, mainly because of the necessary preprocessing time and additional storage requirements.

Actual engineering implementations often use tree structures to guide the point location, for example, the "alternating digital tree" of Bonet and Peraire [4], which typically come very close to the theoretically

\footnotetext{
* Corresponding author. Current address: ANSYS, Inc., 275 Technology Drive, Canonsburg, PA 15317, USA. E-mail: epm@acm.org

${ }^{1}$ Current address: Bios Group LP, 317 Paseo de Peralta, Santa Fe, NM 87501, USA. E-mail: isaac@biosgroup.com.

${ }^{2}$ Current address: City University of Hong Kong, Department of Computer Science, 83 Tat Chee Avenue, Kowloon Tong, Kowloon, Hong Kong. E-mail: bhz@cs.cityu.edu.hk.
} 
optimal logarithmic time complexity of the problem. However, all these methods require a certain amount of preprocessing for the creation of additional data structures (and their maintenance, for the dynamic version of the problem). "Bucketing" algorithms, see, for example, Asano et al. [2], can even achieve constant search time, on average, for uniform distribution, for input in a bounded domain, but they, too, require extra preprocessing, especially within each bucket, and additional storage. Here, we will discuss a technique that is efficient in practice, uses no preprocessing time, no additional storage, and, as a bonus, could not be easier to implement.

Point location, in its full generality, deals with the following problem: given a set of disjoint geometric objects, determine the object containing a query point. The literature often restricts the objects to cells of subdivisions of geometric regions. This work focuses even further on point location within the triangles or tetrahedra of triangulations of 2D and 3D point sets; in fact, the actual analysis is restricted to Delaunay triangulations of random points. The focus on triangulations is justified since regions of arbitrary subdivisions can be triangulated. Moreover, the query problem in triangulations itself occurs quite frequently in practice; for example, in mesh generation and finite-element analysis (FEA).

Delaunay triangulations. For completeness, we briefly include the following definitions. The convex hull of a finite point set $X$ is the smallest convex set containing $X$. The convex hull of a set of $k+1$ affinely independent points in $\mathbb{R}^{d}$, for $0 \leqslant k \leqslant d$, is called a $k$-simplex; that is, a vertex, an edge, a triangle, or a tetrahedron, etc. If $k=d$, we also say the simplex is full dimensional. A triangulation $\mathcal{T}$ of $X$ is a subdivision of the convex hull of $X$ consisting only of simplices with the following two properties: (i) for every simplex in $\mathcal{T}$, all its faces are also simplices in $\mathcal{T}$; (ii) the intersection of any two simplices in $\mathcal{T}$ is either empty or a face of both, in which case it is again a simplex in $\mathcal{T}$. A Delaunay triangulation $\mathcal{D}$ of $X$ is a triangulation in which the circumsphere of each full-dimensional simplex has no points of $X$ in its interior.

Point location by walking. The basic idea is straightforward and not at all new; it goes back to early work on constructing Delaunay triangulations in 2D and 3D [7,18]. Given a Delaunay triangulation $\mathcal{D}$ of a set $X$ of $n$ points in $\mathbb{R}^{d}$, and a target point $q$; in order to locate the (full-dimensional) simplex in $\mathcal{D}$ containing $q$, start at some arbitrary simplex in $\mathcal{D}$ and then "walk" from simplex to neighboring simplex "in the general direction" of the target point $q$. The underlying assumption is that the $\mathcal{D}$ is given by an internal representation allowing constant-time access between neighboring simplices. The list of suitable data structures includes the 2D quad-edge data structure [19], the edge-facet structure in 3D [11], its specialization and compactification to the domain of 3D triangulations [20], or its generalization to $d$ dimensions [8].

A pseudo-code for the simple "walking" method in 2D can be found in [19]. This procedure is guaranteed to terminate only in Delaunay triangulations. For arbitrary triangulations it may go into an infinite loop; see, for example, [15]. However, the infinite loop can be broken, for all practical purposes, by introducing randomness when selecting which neighboring simplex to visit next; see Section 6 .

The "walking" method has been ignored by most theoreticians in computational geometry since not much can be said about its performance theoretically, other than it is "expected" to take time proportional to $n^{1 / d}$ when the points are randomly distributed [18,7]. However, because of its exceptional simplicity, the method is often used by practitioners in the geometric computing community, in particular, in mesh generation for FEA. 
Jump-and-march. In the following, we modify the method somewhat. First, we "march" towards $q$ by strictly traversing the simplices intersected by a line segment $L$, starting at a vertex of the initial simplex, and ending in $q$. This makes it easier to analyze the procedure (and trivially eliminates the above mentioned infinite-loop problem); although, as discussed empirically in Section 6, it seems to reduce the expected number of visited simplices only marginally, and actually degrades the actual performance. Second, we "jump" to a good starting point via random sampling on the point set $\left\{X_{1}, X_{2}, \ldots, X_{n}\right\}$. This enhances the overall procedure significantly; in a sense, we are simulating the effects of the bucketing approach by random sampling.

Given the Delaunay triangulation $\mathcal{D}$ of these $n$ points $\left\{X_{1}, X_{2}, \ldots, X_{n}\right\}$, and a query point $q$, the following procedure locates the simplex of $\mathcal{D}$ containing $q$, if such a simplex exists.

(1) Select $m$ points $Y_{1}, \ldots, Y_{m}$ at random and without replacement from $X_{1}, \ldots, X_{n}$.

(2) Determine the index $j \in\{1, \ldots, m\}$ minimizing the distance $d\left(Y_{j}, q\right)$. Set $Y=Y_{j}$.

(3) Locate the simplex containing $q$ by traversing all simplices intersected by the line segment $(Y, q)$.

Step (3), that is, the straight "march", can be implemented in constant time per simplex visited, once the initial simplex, intersected by $L$ and incident to "starting point" $Y$, is determined.

Motivated by the positive empirical results of [20], where the jump-and-march (or rather, the jumpand-walk; see also Section 6) is used to implement the randomized incremental flip algorithm to construct $3 \mathrm{D}$ Delaunay triangulations, this procedure was recently analyzed for the $2 \mathrm{D}$ case, with the result that the expected query time is $\mathrm{O}\left(n^{1 / 3}\right)$ when the points are randomly distributed [10]. This result, in turn, builds on the work of Bose and Devroye [6] who prove that for any line segment $L$ the expected number of intersected triangles in proportional to $|L| n^{1 / 2}$.

In the following, both results are extended to $\mathbb{R}^{3}$, showing that the jump-and-march point location in spatial Delaunay triangulations of $n$ random points has an expected running time of $\mathrm{O}\left(\delta(n)^{1 / 4} n^{1 / 4}(\log n / \log \log n)^{3 / 4}\right)$, where $\delta(n)$ denotes the expected degree of a Delaunay vertex. A result by Bern et al. [3] on the expected maximum degree would give $\delta(n)=\mathrm{O}(\log n / \log \log n)$. On the other hand, Dwyer [13] shows that $\delta(n)=\mathrm{O}(1)$ for any fixed dimension $d$, under the assumption that the points are chosen uniformly at random in a $d$-dimensional ball. 3D Delaunay triangulations are of quadratic size in the worst-case. Years of "real world" usage in the mesh generation industry seem to suggest, however, that for problems of practical relevance their size is only expected to be linear in $n$. One can further argue then, that $\delta(n)$ is expected to be constant in practice. For the jump-and-march, this means that the expected running time will be close to $\mathrm{O}\left(n^{1 / 4}\right)$. This compares well to the theoretically optimal $\mathrm{O}(\log n)$ bound, at least for practical sizes of input data; for example, $n^{1 / 4} / \log _{2} n<2.5$, for $n$ in the range up to $10^{7}$.

On a theoretical side, our work addresses and solves two difficult issues. First, when proving "probabilistic impossibility results" for Delaunay triangulations one is naturally led to define volumes and to argue that these volumes are likely to contain some Delaunay vertices. One must be careful though to define (as much as possible) these volumes independently from the vertices. We achieve this difficult task in 3D. Second, the perturbing effect of the boundary is very well-known. The probabilistic model of [3], for instance, was designed to analyze typical properties of Delaunay triangulations away from the boundary. Here, we provide a specific estimate of the range of this perturbation. Our methods seem well suited to bring even more precise results.

Outline. The paper is organized as follows. In Sections 2 and 4, we first generalize the result of [6] regarding the intersection of a line segment with a random Delaunay triangulation to $3 \mathrm{D}$. Then, we 
generalize the proof of [10] to 3D. Section 3 presents an outline of the proof. In Sections 5 and 6 , we present empirical results over randomly generated point sets ranging from $n=1000$ to 50000. Our tests confirm that the method is efficient in practice and compares with theoretically optimal $\mathrm{O}(\log n)$ methods, at least in the above range, which seems to be of most relevance for practitioners in GIS and CAD.

\section{Statement of results}

Let $C$ be a convex domain of $\mathbb{R}^{3}$ and let $\alpha$ and $\beta$ be two reals such that $0<\alpha<\beta$. We say that a probability measure $P$ is an $(\alpha, \beta)$-measure over $C$ if $P[C]=1$ and if we have $\alpha \lambda(S) \leqslant P[S] \leqslant \beta \lambda(S)$ for every measurable subset $S$ of $C$, where $\lambda$ is the usual Lebesgue measure. ${ }^{3}$ An $\mathbb{R}^{3}$-valued random variable $X$ is called an $(\alpha, \beta)$-random variable over $C$ if its probability law $\mathcal{L}(X)$ is an $(\alpha, \beta)$-measure over $C$. A particular and important example of an $(\alpha, \beta)$-measure $P$ is when $P$ is a probability measure with density $f(x)$ such that $\alpha \leqslant f(x) \leqslant \beta$ for all $x \in C$. One of the advantages of our more general notion is that it allows for a probability measure charging only points with rational coordinates: this is the case for most computer simulations. This probabilistic model was introduced in [6]. The Poisson model of [3] is related to ours in the sense that, conditioned on the number $n$ of points observed over a finite volume, the probability distribution is uniform, that is, an $(\alpha, \alpha)$-measure.

Below is our main result on the expected running time of the jump-and-march algorithm, when applied on $\mathcal{D}$, the Delaunay triangulation of $n$ random points in $\mathbb{R}^{3}$.

Theorem 1. Let $C$ be a bounded convex set of $\mathbb{R}^{3}$ and let $X_{1}, \ldots, X_{n}$ be $n$ points drawn independently in $C$ from an $(\alpha, \beta)$-measure. Then there exist constants $c_{1}, c_{2}$ and $c_{3}$ depending only upon $\alpha, \beta$ and $C$ such that the following holds. Assume that $m \geqslant n^{1 / 5}$ and that the query point is selected independently of $X_{1}, \ldots, X_{n}$ and is at distance of at least $c_{1} / n^{1 / 18}$ from the boundary $\partial C$. Then the expected time of the jump-and-march algorithm is bounded by

$$
c_{2} m \delta(n)+c_{3}(n / m)^{1 / 3} \log n / \log \log n,
$$

where $\delta(n)$ is the expected vertex degree of the Delaunay triangulation. In particular, the expected time is optimized to $\mathrm{O}\left(\delta(n)^{1 / 4} n^{1 / 4}(\log n / \log \log n)^{3 / 4}\right)$ with the choice of $m=\Theta\left(n^{1 / 4} / \delta(n)^{3 / 4}(\log n /\right.$ $\left.\log \log n)^{3 / 4}\right)$.

The proof of Theorem 1 rests on the following theorem.

Theorem 2. Let $C$ be a bounded convex set of $\mathbb{R}^{3}$ and let $X_{1}, \ldots, X_{n}$ be $n$ points drawn independently in $C$ from an $(\alpha, \beta)$-measure. Then there exist constants $c_{4}$ and $c_{5}$ depending only upon $\alpha, \beta$ and $C$ such that the following holds. Let $L$ be a segment in $C$ being at distance of at least $c_{4}(\log n / n)^{1 / 3}$ from the boundary $\partial C$. Let $N$ be the number of intersections between $L$ and $\mathcal{D}$. Then

$$
E[N] \leqslant c_{5}|L| n^{1 / 3} \log n / \log \log n .
$$

We can easily extend Theorem 2 to the case where $L$ is a random segment independent of the $n$ points $X_{1}, \ldots, X_{n}$. For this, define the event $B=\left\{d(L, \partial C) \geqslant c_{4}(\log n / n)^{1 / 3}\right\}$. We then have $E[N \mid$

\footnotetext{
${ }^{3}$ Note that the relation $\lambda(C) \leqslant 1 / \alpha<\infty$ implies that $C$ has finite area. The convexity of $C$ then implies that $C$ is bounded (that is, that $C$ is included in some finite ball).
} 
$B] \leqslant c_{5} E[|L| \mid B] n^{1 / 3} \log n / \log \log n$. In Section 4, we first prove Theorem 2 following the same ideas as [6]; however, we would like to point out that the technical details are quite different in 3D and more difficult. Given Theorem 2, it is easy to generalize the result of [10] to obtain Theorem 1.

Before presenting the proof let us clarify some rather difficult probabilistic issues underlying our results. It is important to realize that our probability results involve two very different sources of randomness. The first source of randomness is the $(\alpha, \beta)$-measure generating the random input $X_{1}, \ldots, X_{n}$. In particular, the expected vertex degree $\delta(n)$ is computed with respect to this probability measure. The second source of randomness is the randomness introduced in step (1) of our randomized algorithm. The expected values presented in Theorems 1 and 2 are computed over both sources of randomness. In addition, one should realize that, in Theorem 1, the query point is not sampled from any fixed random distribution: this point is chosen non-deterministically. One way to express that, is to say that the query point is selected by an adversary. The adversary can itself use randomness and can be assumed to know the two probability distributions used in our analysis; of course, a critical restriction is that the adversary must select the query point unaware of the realization of these distributions.

To finish, let us mention that the results of Theorems 1 and 2 can be tightened by specifying how the constants $c_{1}, \ldots, c_{5}$ depend on the geometry of $C$. In particular one could show that these constant depend on $C$ only through its volume and the curvature of its boundary.

\section{General outline of the proof}

As mentioned above, Theorem 1 is a rather simple consequence of Theorem 2 . We outline first the proof of Theorem 1. In general, the time required to walk through a segment $L$ is proportional to the number of Delaunay tetrahedra crossed by $L$. By Theorem 2, this number is close to $|L| n^{1 / 3}$ provided that the segment $L$ is chosen independently of the triangulation $\mathcal{D}$. To apply this result to the walk-phase, when one walks from $Y$ (the point selected in the jump-phase) to $q$ (the query point), we proceed as follows. We consider the triangulation $\mathcal{D}^{\prime}$ induced by the $n-m$ points not used in the jump-phase. One can easily show that the total time required by the walk-phase is bounded by the time to walk from $Y$ to $q$ in $\mathcal{D}^{\prime}$, plus the total tetrahedron-degree (in $\mathcal{D}$ ) of the $m$ points $Y_{1}, \ldots, Y_{m}$. The point $Y$ depends only on $Y_{1}, \ldots, Y_{m}$ and is independent from the triangulation $\mathcal{D}^{\prime}$. One can therefore apply Theorem 2 and show that the expected time to walk from $Y$ to $q$ in $\mathcal{D}^{\prime}$ is $\mathrm{O}\left(\left(1 / m^{1 / 3}\right)(n-m)^{1 / 3} \log n / \log \log n\right)$. (The assumption $m \geqslant n^{1 / 5}$ is used there.) On the other hand, the expected tetrahedron-degree (in $\mathcal{D}$ ) of each point $Y_{1}, \ldots, Y_{m}$ is equal to $\delta(n)$. As mentioned in Section $1, \delta(n)=\mathrm{O}(1)$ in practical situations and always at most $\mathrm{O}(\log n / \log \log n)$. This establishes Theorem 1 .

We now turn to an outline of the proof of Theorem 2. This proof is longer and requires several technical lemmas. To bound the (expected) number of tetrahedra traversed by a segment $L$, we cover $L$ with little balls of appropriate radius and reduce the problem to bound the (expected) number $N_{2}$ of tetrahedra intersected by each of these balls $\mathcal{B}(y, r)$. (This idea is directly inspired by [6].) To estimate (the expected value of) $N_{2}$ we introduce and analyze $N_{1}: N_{1}$ is the number of sites $X_{i}$ such that one of the Delaunay tetrahedra incident to $X_{i}$ intersects $\mathcal{B}(y, r)$. To connect $N_{2}$ to $N_{1}$ we use an extension of a result established in [3]: with high probability, for every site $X_{i}$, the number of Delaunay tetrahedra incident to $X_{i}$ is $\mathrm{O}(\log n / \log \log n)$. This allows us to derive that $E\left[N_{2}\right]=\mathrm{O}\left(E\left[N_{1}\right] \log n / \log \log n\right)$.

We have reduced the original problem to the one of estimating an upper bound of $E\left[N_{1}\right]$. For this, we consider a given site $X_{1}$ and estimate the probability that one of the Delaunay tetrahedra incident to 
$X_{1}$ intersects $\mathcal{B}(y, r): E\left[N_{1}\right]$ is equal to $n$ times this probability. Our majoration hinges on a geometric argument: we consider a set of three little balls $A_{1}, A_{2}, A_{3}$ positioned at equal distance between $X_{1}$ and $y$. (This set depends upon $X_{1}$ and $y$ but not upon $X_{2}, \ldots, X_{n}$.) We show that every ball containing $X_{1}$ and intersecting $\mathcal{B}(y, r)$ must contain one of the three balls $A_{1}, A_{2}, A_{3}$. By the Delaunay emptyball condition, no Delaunay tetrahedron incident to $X_{1}$ can intersect $\mathcal{B}(y, r)$ if each of the three balls $A_{1}, A_{2}, A_{3}$ contains a site $X_{j}$. This fact allows us to bound the probability that one of the Delaunay tetrahedra incident to $X_{1}$ intersects $\mathcal{B}(y, r)$. This in turn allows us to bound $E\left[N_{1}\right]$, then $E\left[N_{2}\right]$ and to prove Theorem 2.

To finish we mention some thorny technical issues encountered in the proof. The main customary argument used when proving probabilistic upper bounds for Delaunay triangulations consists of defining volumes (usually balls), and to argue that no Delaunay site should be contained in these volumes. One must be careful to define as much as possible these volumes independently of the Delaunay triangulation. To achieve that purpose we introduce in Section 4.1 the notion of a "spindle": the spindle is composed of the three balls $A_{1}, A_{2}, A_{3}$. The independence of the spindle from the Delaunay triangulation is used critically in the proof of Lemma 5 .

A second difficulty comes from the fact that all three balls $A_{1}, A_{2}, A_{3}$ might not be fully contained in $C$ when $X_{1}$ is too close to the boundary of $C$. The formal treatment of this situation complicates the estimation of $E\left[N_{1}\right]$ done in Lemma 5 .

\section{Probabilistic analysis}

To denote the fact that a simplex $F$ (edge, triangle, or tetrahedron) is part of the Delaunay triangulation $\mathcal{D}$, we write: $F \in \mathcal{D}$. For every four points $x, y, t, z$ in general position we let $\mathcal{B}(x y t z)$ be the unique ball circumscribed to these four points. Also, for every point $x$ and $r \geqslant 0$, we let $\mathcal{B}(x, r)$ denote the ball of radius $r$ centered at point $x$.

Our arguments proceed as follows. First, we state and prove a geometric lemma (Lemma 3), which will later be used in the proof of Lemma 5. Second, we give a set of probabilistic lemmas (Lemmas 4-7), which, in a third step, we combine to prove Theorems 1 and 2.

\subsection{A geometric lemma}

This section is devoted to formally define the construction of the three balls $A_{1}, A_{2}, A_{3}$ and to prove Lemma 3 stating that every ball containing $X_{1}$ and intersecting $\mathcal{B}(y, r)$ must contain one of the three balls $A_{1}, A_{2}, A_{3}$. The construction is based on a geometric object called a spindle, depending on two parameters $k_{1}$ and $k_{2}$. The result of Lemma 3 depends on a third parameter $k_{3}$.

For Lemma 3 we restrict the numbers $k_{1}, k_{2}, k_{3}$ to be positive and such that $k_{1}+k_{3}<\frac{1}{2}$ and $3 k_{2}+2 k_{3}<k_{1}$. In the proof of Lemma 5 we will impose some additional restrictions on the choice of these three numbers.

Let $l$ be a positive number. We define an $l$-spindle to be a geometric object composed of an axle surrounded by three balls:

- The axle is a line segment $x y$ of length $l$.

- At $q=(x+y) / 2$, we attach three spokes of length $k_{1} l$. The spokes are in a plane perpendicular to the axle; each pair of spokes forms an angle of $(2 \pi) / 3$. 


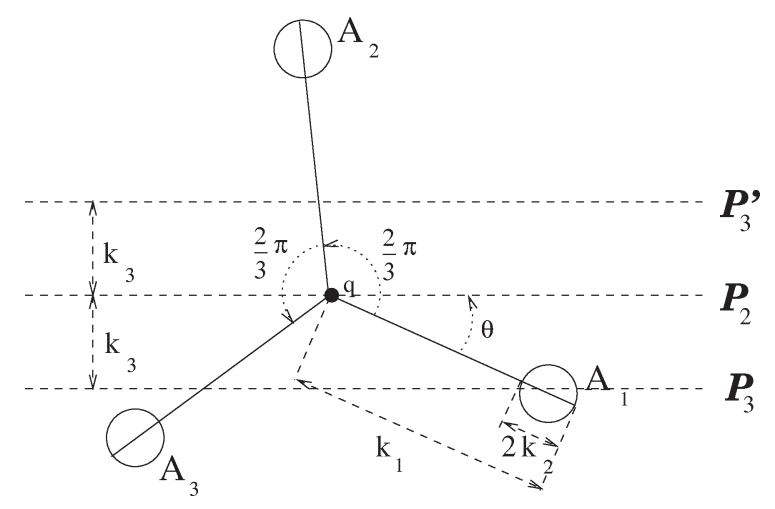

Fig. 1. Defining $k_{1}, k_{2}$ and $k_{3}$. Segment $x y$ is perpendicular to the plane of the figure and has $q$ for its midpoint. The figure sets $l=1$; so $k_{l} l$ becomes $k_{\iota}$ for $\iota=1,2,3$.

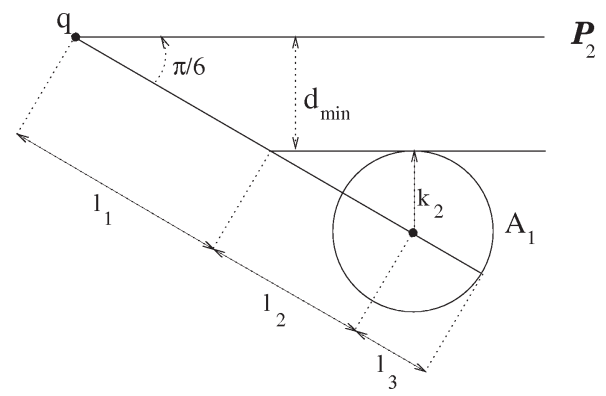

Fig. 2. The case where $\theta=\pi / 6$. Again, we set $l=1$ to simplify.

- We place a ball $A_{i}$ at the end of each of the three spokes $i=1,2,3$. These balls have radius $k_{2} l$ and their centers are at distance $\left(k_{1}-k_{2}\right) l$ from $q$.

The collection of the three balls $A_{1}, A_{2}, A_{3}$ is called the wheel of the spindle.

Fig. 1 illustrates the situation. The plane of the figure (which is the plane of the spindle which contains the centers of $A_{1}, A_{2}, A_{3}$ ) is perpendicular to $x y$ and goes through $q$. Let $\mathcal{P}_{2}$ be any plane going through $q$ and containing $x y$, and let $\mathcal{P}_{3}$ and $\mathcal{P}_{3}^{\prime}$ be the two planes parallel to $\mathcal{P}_{2}$ and at distance $k_{3}$ of $\mathcal{P}_{2}$.

Let also $\theta$ denote the angle between $\mathcal{P}_{2}$ and the spoke of $A_{1}$, and consider the case where $\theta=\pi / 6$. In this case, the balls $A_{1}$ and $A_{3}$ are at the same distance of $\mathcal{P}_{2}$, namely: $d\left(A_{1}, \mathcal{P}_{2}\right)=d\left(A_{3}, \mathcal{P}_{2}\right) \stackrel{\text { def }}{=} d_{\min }$. As illustrated in Fig. $2, d_{\min }=l_{1} \sin (\pi / 6)=l_{1} / 2$. The quantities $l_{1}, l_{2}$ and $l_{3}$ satisfy the three linear relations $l_{1}+l_{2}+l_{3}=k_{1} l$ and $l_{3}=k_{2} l=l_{2} \sin (\pi / 6)$ allowing us to conclude that $d_{\min }=l_{1} / 2=\left(k_{1}-3 k_{2}\right) l / 2$.

For a general value of $\theta$, we can easily check that there is (at least) one ball $A_{i}$ such that $d\left(A_{i}, \mathcal{P}_{2}\right) \geqslant$ $d_{\text {min }}$ and such that $A_{i}$ is on same side of $\mathcal{P}_{2}$ as $\mathcal{P}_{3}$. Symmetrically, there is (at least) one ball $A_{j}$ with $d\left(A_{j}, \mathcal{P}_{2}\right) \geqslant d_{\min }$ and which is on the same side of $\mathcal{P}_{2}$ as $\mathcal{P}_{3}^{\prime}$. Therefore, the choice $k_{3}<d_{\min }$ ensures that, for every value of $\theta$, there are two balls $A_{i}$ and $A_{j}$ such that $A_{i}$ is "below" $\mathcal{P}_{3}$ and such that $A_{j}$ is "above" $\mathcal{P}_{3}^{\prime} .4$

\footnotetext{
4 The terms "below" and "above" are used for simplification and are intuitively made clear by Fig. 1.
} 


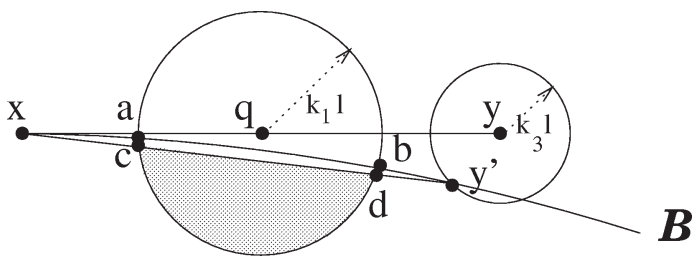

Fig. 3. Plane oqy is the plane of the figure ( $o$, the center of ball $\mathcal{B}$, is not drawn); it is perpendicular to the plane of the spindle $\mathcal{S}$ (that is, the plane of Fig. 1). All the points depicted lie in plane oqy; points $x, a, b, y^{\prime}$ lie on ball $\mathcal{B}$; points $x, c, d$ and $y^{\prime}$ are collinear. The shaded area represents $\mathcal{I}_{2}$.

Lemma 3. Let $x, y, x_{1}, \ldots, x_{n}$ be points in $\mathbb{R}^{3}$ with $l=d(x, y)$ and let $k_{1}, k_{2}, k_{3}$ be three positive numbers such that $k_{1}+k_{3}<\frac{1}{2}$ and $3 k_{2}+2 k_{3}<k_{1}$. Let $\mathcal{S}$ be any $l$-spindle whose axle is $x y$ and let as before $A_{1}, A_{2}, A_{3}$ be the three balls of its wheel. Finally, let $\mathcal{D}$ be the Delaunay triangulation of $x, x_{1}, \ldots, x_{n}$. If all three balls $A_{1}, A_{2}, A_{3}$ each contain a point $x_{j}$, then there exists no tetrahedron in $\mathcal{D}$ incident to $x$ which intersects (touches, crosses or contains) the ball $\mathcal{B}\left(y, k_{3} l\right)$.

Proof. We begin by noting that $k_{1}+k_{3}<\frac{1}{2}$ implies that the balls $\mathcal{B}\left(q, k_{1} l\right)$ and $\mathcal{B}\left(y, k_{3} l\right)$ do not intersect; see also Fig. 3. We are going to show that every ball $\mathcal{B}$ having $x$ on its boundary and intersecting ball $\mathcal{B}\left(y, k_{3} l\right)$ must contain one of the three balls $A_{1}, A_{2}, A_{3}$ in its interior. This implies the lemma: assume there exists a tetrahedron $x t z w \in \mathcal{D}$ intersecting $\mathcal{B}\left(y, k_{3} l\right)$. The ball $\mathcal{B}(x t z w)$ circumscribed to $x t z w$ obviously also intersects $\mathcal{B}\left(y, k_{3} l\right)$. The prerequisite of the lemma states that each of the $A_{1}, A_{2}, A_{3}$ contains a point $x_{j}$. As we claim, this implies that $\mathcal{B}(x t z w)$ contains one of the $A_{1}, A_{2}, A_{3}$ and thus the corresponding $x_{j}$. This contradicts the assumption that $x t z w$ is a Delaunay tetrahedron.

Consider therefore a ball $\mathcal{B}$ having $x$ on its boundary and intersecting $\mathcal{B}\left(y, k_{3} l\right)$; let $o$ denote its center. Fig. 3 illustrates the situation as a projection onto the plane $o q y$, which is perpendicular to the plane of $\mathcal{S}$. All the points mentioned in the figure do belong to the plane oqy (but $o$ is not represented for lack of space). In particular, the point $x$, being on the line $q y$, is part of the figure. Also, let $y^{\prime}$ be a point of $\mathcal{B} \cap \mathcal{B}\left(y, k_{3} l\right) \cap$ Plane $($ oqy $) .{ }^{5}$

The radius $k_{3} l$ being smaller than the radius $k_{1} l$, the line $x y^{\prime}$ necessarily intersects $\mathcal{B}\left(q, k_{1} l\right)$; see Fig. 3. The intersection is a segment that we denote $c d$. By convexity, the ball $\mathcal{B}$ contains $x y^{\prime}$ and hence also contains $c d$. This immediately implies that the intersection of the two balls $\mathcal{B} \cap \mathcal{B}\left(q, k_{1} l\right) \stackrel{\text { def }}{=} \mathcal{I}_{1}$ is not empty. $\mathcal{I}_{1}$ is therefore either one of the two balls or the union of two spherical caps. The fact that $k_{1} \leqslant k_{3}+k_{1}<1 / 2$ implies that $x$ does not belong to $\mathcal{B}\left(q, k_{1} l\right)$ and hence that $\mathcal{I}_{1} \neq \mathcal{B}$. On the other hand, if $\mathcal{I}_{1}=\mathcal{B}\left(q, k_{1} l\right)$ then $\mathcal{B}$ contains all three balls $A_{1}, A_{2}, A_{3}$ and we are done.

We can therefore reduce the analysis to the case of Fig. 3: the intersection $\mathcal{I}_{1}$ (is convex and) consists of two spherical caps sitting on a disk $\mathcal{C}_{1}$; this disk is perpendicular to the line segment $o q$ (joining the centers of the two balls) and hence perpendicular to the plane oqy of Fig. 3; the latter plane intersects $\mathcal{C}_{1}$ in the line segment $a b$. Consider now another plane, also perpendicular to the plane oqy, but containing the line segment $x y^{\prime}$. This plane cuts $\mathcal{B}\left(q, k_{1} l\right)$ in a disk that we denote $\mathcal{C}_{2}$. This disk is also perpendicular to the plane oqy which it intersects in line segment $c d$. Fig. 3 illustrates that, by convexity of the arc $x a b y^{\prime}$, the line segment $x c d y^{\prime}$ does not intersect the segment $a b$. This implies that the two disks $\mathcal{C}_{1}$ and $\mathcal{C}_{2}$ (which are both perpendicular to plane $o q y$ ) do not intersect. Now set $\mathcal{I}_{2}$ to be the spherical cap of

\footnotetext{
${ }^{5}$ This intersection is not empty, because $\mathcal{B}$ intersects $\mathcal{B}\left(y, k_{3} l\right)$.
} 


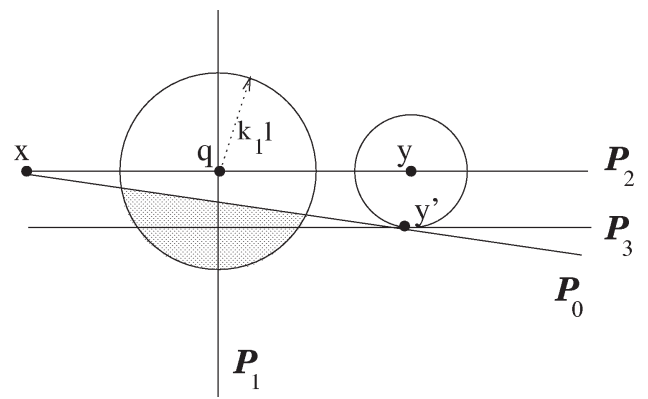

Fig. 4. The minimal spherical cap $\mathcal{I}_{2}$ cut out of the ball $\mathcal{B}\left(q, k_{1} l\right)$ by the plane $\mathcal{P}_{0}$ containing $x$ and tangent to $\mathcal{B}\left(y, k_{3} l\right)$. The plane of the picture is the plane oqy, where $o$ is the center of $\mathcal{B}$.

$\mathcal{B}\left(q, k_{1} l\right)$ "below" disk $\mathcal{C}_{2}$, that is, the spherical cap of $\mathcal{B}\left(q, k_{1} l\right)$ cut by $\mathcal{C}_{2}$ which does not contain the disk $\mathcal{C}_{1}$. Our discussion allows us to conclude that $\mathcal{I}_{2} \subseteq \mathcal{I}_{1}$.

Recall that, in the previous argument, $y^{\prime}$ is any point in $\mathcal{B} \cap \mathcal{B}\left(q, k_{3} l\right) \cap$ Plane (oqy). Therefore, in the case where $y$ belongs to $\mathcal{B}$ we can select $y^{\prime}$ to be equal to $y$. In that case $\mathcal{I}_{2}$ is an hemisphere of $\mathcal{B}\left(q, k_{1} l\right)$ (which contains trivially one of the balls $A_{1}, A_{2}, A_{3}$ ). On the other hand, if $y \notin \mathcal{B}$, then $\mathcal{I}_{2}$ is the smaller of the two spherical caps determined by $\mathcal{C}_{2}$.

Summarizing all the previous discussion, we have established so far that, if $\mathcal{B}$ is a ball having $x$ on its boundary and intersecting $\mathcal{B}\left(y, k_{3} l\right)$, then there exists a plane $\mathcal{P}$ containing $x$, intersecting $\mathcal{B}\left(y, k_{3} l\right)$, and such that the intersection $\mathcal{B} \cap \mathcal{B}\left(q, k_{1} l\right)$ contains the smaller of the two spherical caps cut out of $\mathcal{B}\left(q, k_{1} l\right)$ by $\mathcal{P}$. Furthermore, it is clear that we can restrict ourselves to the case where $\mathcal{P}$ is tangent to $\mathcal{B}\left(y, k_{3} l\right)$; this corresponds to the case where the spherical cap $\mathcal{I}_{2}$ is minimal among all spherical caps cut by planes containing $x$ and intersecting $\mathcal{B}\left(y, k_{3} l\right)$. We can therefore rewrite the preceding summary as follows: Let $\mathcal{B}$ be any ball having $x$ on its boundary and intersecting $\mathcal{B}\left(y, k_{3} l\right)$. Then there exists a plane $\mathcal{P}_{0}$ containing $x$ and tangent to $\mathcal{B}\left(y, k_{3} l\right)$ such that the intersection $\mathcal{B} \cap \mathcal{B}\left(q, k_{1} l\right)$ contains the smaller of the two spherical caps cut out of $\mathcal{B}\left(q, k_{1} l\right)$ by $\mathcal{P}$.

Fig. 4 illustrates the new situation. $\mathcal{P}_{0}$ is such a plane tangent to $\mathcal{B}\left(y, k_{3} l\right)$, and $\mathcal{I}_{2}$ is the smaller of the two spherical caps that $\mathcal{P}_{0}$ cuts off $\mathcal{B}\left(q, k_{1} l\right)$. Now let $\mathcal{P}_{2}$ be the plane perpendicular to the plane oqy (the plane of the figure) which contains the line $x q y$, and let $\mathcal{P}_{3}$ be the plane parallel to $\mathcal{P}_{2}$ and tangent to $\mathcal{B}\left(y, k_{3} l\right)$; obviously, the distance from $q$ to $\mathcal{P}_{3}$ is equal to $k_{3} l$. Finally, let $\mathcal{P}_{1}$ denote the plane that contains $q$ and is perpendicular to $\mathcal{P}_{2}$. Note that $\mathcal{P}_{1}$ is also the plane of Fig. 1 defined by the centers of the three balls $A_{1}, A_{2}$ and $A_{3}$ of the spindle $\mathcal{S}$. Moreover, the planes $\mathcal{P}_{2}$ and $\mathcal{P}_{3}$ intersect $\mathcal{P}_{1}$ as indicated on Fig. 1.

To come to a conclusion, let $\mathcal{I}_{3}$ denote the smaller of the two spherical caps cut by $\mathcal{P}_{3}$ into $\mathcal{B}\left(q, k_{1} l\right)$. Our earlier choices for $k_{1}, k_{2}, k_{3}$ imply that $\mathcal{I}_{3}$ contains at least one of the three balls $A_{1}, A_{2}, A_{3}$ of the spindle $\mathcal{S}$. Since $\mathcal{I}_{3} \subseteq \mathcal{I}_{2} \subseteq \mathcal{I}_{1}$, we established that the ball $\mathcal{B}$ contains at least one of the three balls of the spindle, and Lemma 3 is proven.

\subsection{A set of probabilistic lemmas}

In order to prove the theorems, we need a set of probabilistic lemmas. The proof of Lemma 5 hinges critically on Lemma 3. 


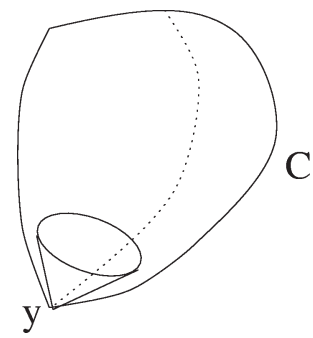

Fig. 5. For every point $y$ of a convex solid $C$ and every small $r, C$ contains a spherical cone of radius $r$ and of solid angle $\theta$ issued from $y$.

Lemma 4. Let $X_{1}$ be a random variable drawn from an $(\alpha, \beta)$-measure over a bounded convex set $C$. Then there exist constants $r_{0}>0$ and $\gamma>0$ such that for every $r \leqslant r_{0}$,

$$
\inf _{y \in C} P\left[d\left(X_{1}, y\right) \leqslant r\right] \geqslant \gamma r^{3} .
$$

Proof. By convexity of $C$ there exists a distance $r_{0}$ and a value $\theta \leqslant 4 \pi$ such that the following holds. Let $y$ be any point in $C$. Then there exists a spherical cone $\mathcal{C}$ with summit $y$, radius $r$ and solid angle $\theta$ such that $\mathcal{C}$ is included in $C$; see Fig. 5. The volume $\operatorname{Vol}(\mathcal{C})$ of this cone is $(\theta / 4 \pi) r^{3}$. Therefore, if $X_{1}$ is a random variable drawn from an $(\alpha, \beta)$-measure over $C$, we have

$$
P\left[d\left(X_{1}, y\right) \leqslant r\right] \geqslant P\left[X_{1} \in \mathcal{C}\right] \geqslant \alpha \operatorname{Vol}(\mathcal{C})=\alpha \frac{\theta}{4 \pi} r^{3} .
$$

In the following, we denote the set of all Delaunay tetrahedra incident to a point $X_{i}$ by $\mathcal{T}_{X_{i}}$. Lemma 5 estimates $N_{1}$, the number of $X_{i}$ 's such that $\mathcal{T}_{X_{i}}$ intersects $\mathcal{B}(y, r)$; in other words, the number of $X_{i}$ 's with the property that at least one of its incident Delaunay tetrahedra intersects $\mathcal{B}(y, r)$. With this, Lemma 6 will estimate the number of Delaunay tetrahedra intersecting $\mathcal{B}(y, r)$.

Lemma 5. Consider $n$ points $X_{1}, \ldots, X_{n}$ drawn independently from an $(\alpha, \beta)$-measure over a bounded convex set $C$. Then there exist positive constants $a, b, c$ and $d$, depending upon $\alpha, \beta$ and $C$ only, such that the following holds. Let $y$ be any point in $C$ at distance $a(\log n / n)^{1 / 3}$ from the boundary $\partial C$ and $r \leqslant 1 / 2 d(y, \partial C)$ be a positive quantity. Let $N_{1}$ denote the number of $X_{i}$ 's with the property that one of the tetrahedra in $\mathcal{T}_{X_{i}}$ intersects $\mathcal{B}(y, r)$. Then

$$
E\left[N_{1}\right] \leqslant b+c r^{2} n^{2 / 3}+d n r^{3} .
$$

Proof. By linearity of the expectation, $E\left[N_{1}\right]=n p$, where $p$ is the probability that one of the Delaunay tetrahedra incident to $X_{1}$ intersects $\mathcal{B}(y, r)$. Let $L=d\left(X_{1}, y\right) ; L$ is itself a random variable. In the following we condition on the value of $X_{1}$. The points $X_{1}$ and $y$ are then fixed, and we let $\mathcal{S}_{X_{1}}$ be any $L$-spindle whose axle is $X_{1} y$. (Hence the angle $\theta$ of Fig. 1 is arbitrary but fixed.) As before, $A_{1}, A_{2}, A_{3}$ denote the 3 balls of the wheel. Remark that the event $\left\{\mathcal{S}_{X_{1}} \subseteq C\right\}=\left\{\right.$ the 3 balls of the spindle $\mathcal{S}_{X_{1}}$ are included in $C$ \}.

In the following, let event $B_{1}=\left\{\mathcal{S}_{X_{1}} \subseteq C, k_{3} L \geqslant r\right\}$. Note that the relation $k_{3} L \geqslant r$ implies that $X_{1}$ does not belong to $\mathcal{B}(y, r)$. The first inequality is therefore a direct consequence of Lemma 3 . In the third inequality, $\mathbf{1}_{k_{2} L \leqslant r_{0}}$ denotes the random variable equal to 1 when $k_{2} L \leqslant r_{0}$ and 0 else. 


$$
\begin{aligned}
P & {\left[\mathcal{T}_{X_{1}} \text { intersects } \mathcal{B}(y, r) \mid X_{1}, B_{1}\right] } \\
& \leqslant P\left[\text { one of the } 3 \text { balls } A_{1}, A_{2}, A_{3} \text { contains no point } X_{2}, \ldots, X_{n} \mid X_{1}, B_{1}\right] \\
& \leqslant \sum_{j=1}^{3} P\left[A_{j} \text { contains none of the points } X_{2}, \ldots, X_{n} \mid X_{1}, B_{1}\right] \\
& =\sum_{j=1}^{3} \prod_{i=2}^{n} P\left[X_{i} \notin A_{j} \mid X_{1}, B_{1}\right] \\
& \leqslant 3 E\left[\left(1-\gamma\left(k_{2} L\right)^{3}\right)^{n-1} \mathbf{1}_{k_{2} L \leqslant r_{0}} \mid X_{1}, B_{1}\right]+3 E\left[\left(1-\gamma r_{0}^{3}\right)^{n-1} \mathbf{1}_{k_{2} L>r_{0}} \mid X_{1}, B_{1}\right] .
\end{aligned}
$$

Eq. (2) is a consequence of the (conditional) independence of the events $X_{i} \notin A_{j}$. This independence comes both from the fact that the random variables $X_{i}$ are independent and the fact that the 3 balls $A_{1}, A_{2}, A_{3}$ are defined independently of the points $X_{2}, \ldots, X_{n}$. The necessity of this last independence is not always recognized and leads to frequent mistakes in the literature.

We now justify the last inequality. Note first that, by Lemma $4, \gamma\left(k_{2} L\right)^{3} \leqslant 1$ when $k_{2} L \leqslant r_{0}$. The expression $\left(1-\gamma\left(k_{2} L\right)^{3}\right)^{n-1}$ is therefore well-defined. The conditioning on $\mathcal{S}_{X_{1}} \subseteq C$ ensures that each ball $A_{i}$ is fully contained in $C$. This implies in particular that the center $y_{i}$ of each ball $A_{i}$ is in $C$. We can then apply Lemma 4 (which determines the values $\gamma$ and $r_{0}$ ) using the fact that the random variables $X_{i}$ are all drawn independently according to an $(\alpha, \beta)$-measure.

We let $\overline{B_{1}}=\left\{k_{3} L<r\right\} \cup\left\{\mathcal{S}_{X_{1}} \nsubseteq \subset C\right\}$ denote the complement of $B_{1}$. Integrating the previous majoration of $P\left[\mathcal{T}_{X_{1}}\right.$ intersects $\left.\mathcal{B}(y, r) \mid X_{1}, B_{1}\right]$ with respect to $X_{1}$ therefore gives

$$
\begin{aligned}
p= & P\left[\mathcal{T}_{X_{1}} \text { intersects } \mathcal{B}(y, r), \overline{B_{1}}\right]+P\left[\mathcal{T}_{X_{1}} \text { intersects } \mathcal{B}(y, r), B_{1}\right] \\
\leqslant & P\left[k_{3} L<r\right]+P\left[\mathcal{T}_{X_{1}} \text { intersects } \mathcal{B}(y, r), \mathcal{S}_{X_{1}} \not C\right]+3 E\left[\left(1-\gamma\left(k_{2} L\right)^{3}\right)^{n-1} \mathbf{1}_{k_{2} L \leqslant r_{0}} \mathbf{1}_{k_{3} L \geqslant r}\right] \\
& +3\left(1-\gamma r_{0}^{3}\right)^{n-1} P\left[k_{2} L>r_{0} ; k_{3} L \geqslant r\right] \\
& \stackrel{\text { def }}{=} \mathrm{I}+\mathrm{II}+\mathrm{III}+\mathrm{IV} .
\end{aligned}
$$

The fact that $X_{1}$ is drawn from an $(\alpha, \beta)$-measure implies that $I=P\left[k_{3} L<r\right] \leqslant(4 / 3) \beta \pi\left(r / k_{3}\right)^{3}$. Also IV $\leqslant 3 \mathrm{e}^{-(n-1) \gamma r_{0}^{3}}$, which is exponentially small with $n$ sufficiently large. We now turn to III. Note first that $\left(1-\gamma\left(k_{2} L\right)^{3}\right)^{n-1} \mathbf{1}_{k_{2} L \leqslant r_{0}} \leqslant \mathrm{e}^{-(n-1) \gamma\left(k_{2} L\right)^{3}}$ and therefore, III $\leqslant 3 E\left[\mathrm{e}^{-(n-1) \gamma\left(k_{2} L\right)^{3}} \mathbf{1}_{k_{3} L \geqslant r}\right]$. To estimate this expression we use spherical coordinates and obtain

$$
\mathrm{III} \leqslant 16 \pi^{2} \beta\left(\frac{2}{3(n-1) k_{2}^{3} \gamma}+\frac{2 r^{2}}{k_{2} k_{3}^{2}(\gamma(n-1))^{1 / 3}} \frac{1}{3} \Gamma\left(\frac{1}{3}\right)\right) .
$$

We now turn to expression II $=P\left[\mathcal{T}_{X_{1}}\right.$ intersects $\left.\mathcal{B}(y, r), \mathcal{S}_{X_{1}} \nsubseteq C\right]$; its majoration will involve showing that only vertices $X_{1}$ within distance $\mathrm{O}\left((\log n / n)^{1 / 3}\right)$ of $y$ have a Delaunay tetrahedron extending to $y$. Furthermore, the condition $\mathcal{S}_{X_{1}} \nsubseteq C$ implies that the spindle is in the vicinity of the boundary $\partial C$ of $C$, which appears to be flat at the very small distance $\mathrm{O}\left((\log n / n)^{1 / 3}\right)$ that we consider. This allows us to model $\partial C$ locally to be a plane $\mathcal{P}: C$ appears locally like a half-plane $U$.

To simplify we set $K=2 k_{1}$ and recall that $K<1-2 k_{3}<1$. Recall also that we defined $\mathcal{S}_{X_{1}}$ to be any arbitrary, externally fixed, spindle whose axle is $y X_{1}: \mathcal{S}_{X_{1}}$ is not uniquely determined by $X_{1}$ but also by the angle $\theta$ of Fig. 1 . For every $X_{1}$ define $\mathcal{S}_{X_{1}}^{\prime}$ to be the 3 -dimensional "tire" span by $\mathcal{S}_{X_{1}}$ when rotating 


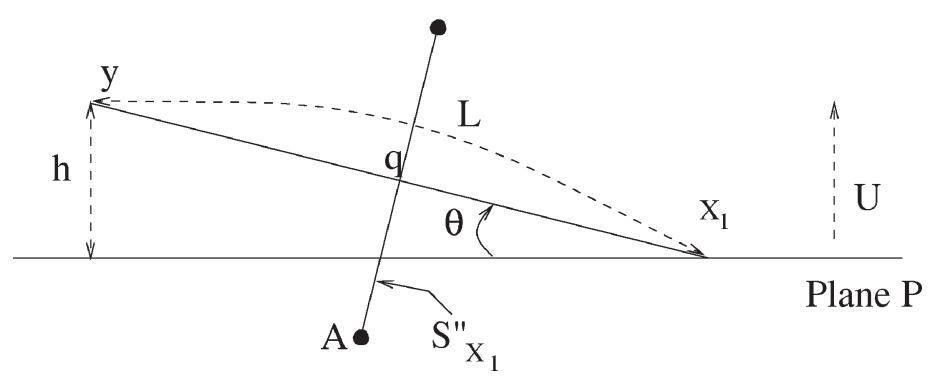

Fig. 6. A spindle $\mathcal{S}_{x_{0}}^{\prime \prime}$ crossing $\mathcal{P}$.

around its axle. $\mathcal{S}_{X_{1}}^{\prime}$ is uniquely determined by $X_{1}$ (along with $y, K$ ) and contains $\mathcal{S}_{X_{1}}$ so that, clearly, $\left\{\mathcal{S}_{X_{1}} \nsubseteq U\right\} \subseteq\left\{\mathcal{S}_{X_{1}}^{\prime} \nsubseteq U\right\}$. To further simplify, introduce $\mathcal{S}_{X_{1}}^{\prime \prime}$ to be the following (simpler) object. $\mathcal{S}_{X_{1}}^{\prime \prime}$ is composed of (i) the axle $X_{1} y$, and (ii) a circle of diameter $K$, the wheel, perpendicular to $X_{1} y$ whose center is the mid-point $q$ of $X_{1} y$. We sometimes write $\mathcal{S}_{X_{1}}^{\prime \prime}(K)$ to emphasize the value of $K$. Furthermore, let $\mathcal{P}$ be a plane such that $y \notin \mathcal{P}$, let $U$ denote the $\mathcal{P}$-half-space to which $y$ belongs, and let $h$ denote the distance $d(y, \mathcal{P})$ from $y$ to $\mathcal{P}$. We only consider points $X$ in $U$ and say that the spindle $\mathcal{S}_{X_{1}}^{\prime \prime}$ crosses $\mathcal{P}$ if its wheel crosses $\mathcal{P}$. As before, we set $L=d\left(X_{1}, y\right)$.

\section{Claim.}

(a) $\mathcal{S}_{X_{1}}^{\prime \prime}(K)$ crosses $\mathcal{P}$ only if $L \geqslant L_{0}(h) \stackrel{\text { def }}{=} \sqrt{1+1 / K^{2}} h$.

(b) If $K \leqslant 0.2$ then $\left\{\mathcal{S}_{X_{1}}^{\prime}(K) \nsubseteq U\right\} \subseteq\left\{\mathcal{S}_{X_{1}}^{\prime \prime}(1.02 K) \nsubseteq U\right\}$.

We just show (a). Assertion (b) implies that, for $k_{1}$ small enough, a minute adjustment in $k_{1}$ allows to consider $\left\{\mathcal{S}_{X_{1}}^{\prime \prime} \nsubseteq U\right\}$ in place of $\left\{\mathcal{S}_{X_{1}}^{\prime} \nsubseteq U\right\}$.

Consider a given value of $L$ and assume that an $L$-spindle $\mathcal{S}_{x_{1}}^{\prime \prime}$ crosses $\mathcal{P}$. Let $m$ be a point on the wheel "below" $\mathcal{P}$; see Fig. 6 . Note that $k_{1}<1 / 2$ implies that $d(y, \mathcal{P}) \leqslant d(y, m)=\left(d(y, q)^{2}+d(q, m)^{2}\right)^{1 / 2}<$ $L / \sqrt{2}$. This implies that there exists a point $x_{0} \in \mathcal{P}$ and such that $d\left(y, x_{0}\right)=L$. (This point can be obtained by rotating the spindle around $y$.) Consider $\mathcal{S}_{x_{0}}^{\prime \prime}$, the spindle associated to $x_{0}$. The fact that $\mathcal{S}_{x_{1}}^{\prime \prime}$ crosses $\mathcal{P}$ implies that $\mathcal{S}_{x_{0}}^{\prime \prime}$ similarly crosses $\mathcal{P}$. (We provide a pictorial justification of this fact. Consider the rotation of the spindle around $y$ in the plane of Fig. 6: we let $\phi$ denote the angle between $y m$ and the vertical line; $\phi_{1}$ is the angle corresponding to the initial point $x_{1} ; \phi_{0}$ is the angle corresponding to $x_{0}$; we also let $m_{1}$ and $m_{0}$ denote the positions of $m$ associated respectively to $x_{1}$ and $x_{0}$. Obviously, $m$ goes "deeper" below $\mathcal{P}$ as $\phi \in[0, \pi]$ decreases to 0 . As $d(y, q)>d(q, m)$ the angle $\phi_{0}$ belongs to $[0, \pi / 2]$. Therefore $m_{0}$ is deeper below $\mathcal{P}$ then $m_{1}$.)

To prove impossibility results we can therefore restrict ourselves to $x \in \mathcal{P}$. Consider such an $x$. The fact that $\mathcal{S}_{x}^{\prime \prime}$ crosses $\mathcal{P}$ implies that $d(q, m)=k_{1} L \geqslant L / 2 \tan \theta$ where $\theta=\arcsin (h / L)$. This immediately implies $L^{2} \geqslant\left(\left(1+K^{2}\right) / K^{2}\right) h^{2}$. We are now ready for the majorations below.

$$
\begin{aligned}
& P\left[\mathcal{S}_{X_{1}}^{\prime \prime} \nsubseteq U, \mathcal{T}_{X_{1}} \text { intersects } \mathcal{B}(y, r)\right] \\
& \quad=P\left[\mathcal{S}_{X_{1}}^{\prime \prime} \nsubseteq U, \exists X_{i_{2}}, X_{i_{3}}, X_{i_{4}} \text { such that } X_{1} X_{i_{2}} X_{i_{3}} X_{i_{4}} \in \mathcal{D}\right. \\
& \left.\quad \quad \text { and such that } X_{1} X_{i_{2}} X_{i_{3}} X_{i_{4}} \cap \mathcal{B}(y, r) \neq \emptyset\right] \\
& \quad \leqslant \max _{y^{\prime} \in \mathcal{B}(y, r)} P\left[\exists X_{i_{2}}, X_{i_{3}}, X_{i_{4}} ; d\left(X_{1}, y\right) \geqslant L_{0}(h), X_{1} X_{i_{2}} X_{i_{3}} X_{i_{4}} \in \mathcal{D}, y^{\prime} \in \mathcal{B}\left(X_{1} X_{i_{2}} X_{i_{3}} X_{i_{4}}\right)\right]
\end{aligned}
$$




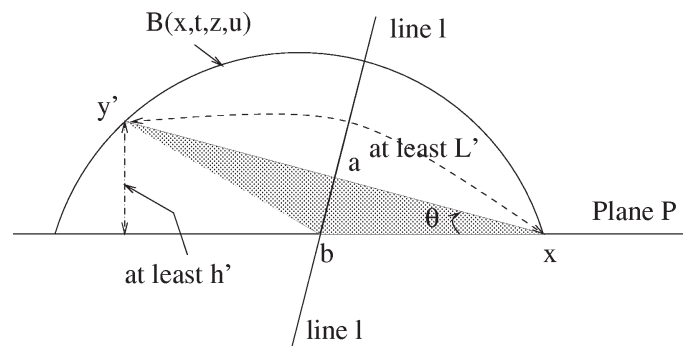

Fig. 7. The cone $\mathcal{C}$ contained in every $\mathcal{B}(x t z u)$, containing $y^{\prime}$ and such that $d\left(x, y^{\prime}\right) \geqslant L_{0}(h)$.

$$
\begin{aligned}
& \leqslant \max _{y^{\prime} \in \mathcal{B}(y, r)}\left(\begin{array}{c}
n-1 \\
3
\end{array}\right) P\left[d\left(X_{1}, y\right) \geqslant L_{0}(h), X_{1} X_{2} X_{3} X_{4} \in \mathcal{D}, y^{\prime} \in \mathcal{B}\left(X_{1} X_{2} X_{3} X_{4}\right)\right] \\
& \leqslant \max _{y^{\prime} \in \mathcal{B}(y, r)} \frac{n^{3}}{6} \int_{T_{y^{\prime}}} \mathrm{d} P_{X_{1}, X_{2}, X_{3}, X_{4}}(x, t, z, u)\left(P\left[X_{5} \notin \mathcal{B}(x t z u)\right]^{n-4}\right),
\end{aligned}
$$

where $T_{y^{\prime}} \stackrel{\text { def }}{=}\left\{(x, t, z, u) \in U^{4} ; d(x, y) \geqslant L_{0}(h)\right.$ and $\left.y^{\prime} \in \mathcal{B}(x t z u)\right\}$, and $P_{X_{1}, X_{2}, X_{3}, X_{4}}$ denotes the probability law of the random variable $\left(X_{1}, X_{2}, X_{3}, X_{4}\right)$.

We now compute an upper-bound for the expression $P\left[X_{5} \notin \mathcal{B}(x t z u)\right]$ when the points $x, t, z, u$ of $U$ are such that $d(x, y) \geqslant L_{0}(h)$, and such that $y^{\prime} \in \mathcal{B}(x t z u)$. We define $K^{\prime} \stackrel{\text { def }}{=} \sqrt{1+1 / K^{2}}$. Hence $L_{0}(h)=K^{\prime} h$. We also set $K^{\prime \prime} \stackrel{\text { def }}{=} 2 K^{\prime}-1$. We need the following claim.

Claim. If $d(x, y) \geqslant L_{0}(h)$ and $y^{\prime} \in \mathcal{B}(x t z u) \cap B(y, r)$ then

$$
\operatorname{Vol}(\mathcal{B}(x t z u) \cap U) \geqslant \frac{\pi}{24}\left(\frac{K^{\prime \prime 2}}{1-1 / K^{\prime \prime 2}}\right)^{1 / 2} h^{3} \stackrel{\text { def }}{=} c^{\prime} h^{3} \text {. }
$$

The situation is presented in Fig. 7. The ball $\mathcal{B}(x t z u)$ cuts minimally $U$ only (i) when $x$ is on the boundary $\mathcal{P}$, (ii) when $y^{\prime}$ is on the boundary of $\mathcal{B}(x t z u)$, and (iii) when the center $o$ of $\mathcal{B}(x t z u)$ is such that the plane $x o y^{\prime}$ is perpendicular to $\mathcal{P}$. We therefore consider the situation within plane $x o y^{\prime}$, as in Fig. 7.

The center $o$ is located on the line $l$ perpendicular to $y^{\prime} x$ and going through the mid-point $a$ of $y^{\prime} x$. Let $b$ be the intersection of $l$ with $\mathcal{P}$. Consider the cone $\mathcal{C}$ issued from $b$ and whose base is the circle with diameter $y^{\prime} x$. We claim that $\mathcal{C}$ is included in the ball $\mathcal{B}(x t z u)$ cutting minimally $U$. (i) This ball $\mathcal{B}(x t z u)$ must have its center $o$ below $a$ : if not, at least half of $\mathcal{B}(x t z u)$ is in $U$. (ii) If $o$ is under $b$, then $b$ is in the convex hull of the 3 points $y^{\prime}, x, o$. All 3 points $y^{\prime}, x, o$ are in $\mathcal{B}(x t z u)$ and hence so is $b$. $\mathcal{B}(x t z u)$ then clearly contains $\mathcal{C}$. (iii) If $c$ belongs to the segment $a b$ then $d(o, b) \leqslant k_{1} L<L / 2 \leqslant d\left(o, y^{\prime}\right)$. Thus, if $o \in[a, b]$ then $b \in \mathcal{B}(x t z u)$ and $\mathcal{B}(x t z u)$ contains $\mathcal{C}$ as before.

This shows that $v \stackrel{\text { def }}{=} \operatorname{Vol}(\mathcal{B}(x t z u) \cap U) \geqslant \operatorname{Vol}(\mathcal{C})$. Using the fact that $d\left(y, y^{\prime}\right) \leqslant r \leqslant h / 2$, we find that $v$ is at least equal to $(\pi / 3)\left(L^{\prime} / 2\right)^{3} \tan \left(\theta^{\prime} / 2\right)$, where $h^{\prime} \stackrel{\text { def }}{=} h-h / 2=h / 2, L^{\prime} \stackrel{\text { def }}{=} L_{0}(h)-h / 2=K^{\prime \prime} h^{\prime}$ and where $\theta^{\prime}=\arcsin \left(h^{\prime} / L^{\prime}\right)$. We obtain $v \geqslant c^{\prime} h^{3}$, where

$$
c^{\prime}=\frac{\pi}{24}\left(\frac{K^{\prime \prime 2}}{1-1 / K^{\prime \prime 2}}\right)^{1 / 2}
$$

and have thus proven 


$$
P\left[\mathcal{S}_{X_{1}}^{\prime \prime} \nsubseteq C, \mathcal{T}_{X_{1}} \text { intersects } \mathcal{B}(y, r)\right] \leqslant \frac{n^{3}}{6}\left(1-c^{\prime} \alpha h^{3}\right)^{n-4} \leqslant \frac{n^{3}}{6} \mathrm{e}^{-(n-4) c^{\prime} \alpha h^{3}} .
$$

Selecting

$$
h(n)=\left(\frac{4}{c^{\prime} \alpha} \frac{\log n}{n}\right)^{1 / 3}
$$

gives II $=\mathrm{O}(1 / n)$, as needed. Then, multiplying by $n$ the bounds found for I, II, III and IV and summing establishes the result, and concludes the proof of Lemma 5.

Lemma 6. Under the hypothesis of Lemma 5,

$$
E\left[N_{2}\right] \leqslant e\left(b+c r^{2} n^{2 / 3}+d n r^{3}\right) \log n / \log \log n,
$$

where $\mathrm{N}_{2}$ is the number of Delaunay tetrahedra that intersect $\mathcal{B}(y, r)$ and where $e$ is a constant depending solely on $C, \alpha$ and $\beta$.

To prove this, we need the following result, very similar to the result derived in [3, Theorem 7].

Lemma 7. There exists a constant $c^{\prime \prime}$ such that $P\left[d^{o}\left(X_{1}\right)>c^{\prime \prime} \log n / \log \log n\right] \leqslant 1 / n^{4}$. Therefore,

$$
P\left[\exists i, d^{o}\left(X_{i}\right)>c^{\prime \prime} \log n / \log \log n\right] \leqslant 1 / n^{3} .
$$

Proof. The proof of this result follows very closely the proof for the Poisson model given in [3, Lemmas 8 and 9]. The only technical difference is that they bound the first probability by $1 / n^{2}$ instead of $1 / n^{4}$. We show here that $1 / n^{4}$ is similarly valid. A careful reading of their proof shows that we only need to establish that, with probability at least $1-1 / n^{4}$, the maximum Delaunay edge length is $\mathrm{O}\left((\log n / n)^{1 / 3}\right) .^{6}$

We compute

$$
\begin{aligned}
P\left[d\left(X_{1}, X_{2}\right) \geqslant h ; X_{1} X_{2} \in \mathcal{D}\right] & \leqslant P\left[d\left(X_{1}, X_{2}\right) \geqslant h ; \exists i_{3}, i_{4} \text { such that } X_{1} X_{2} X_{i_{3}} X_{i_{4}} \in \mathcal{D}\right] \\
& \leqslant n^{2}\left(1-\gamma(h / 2)^{3}\right)^{n-d-1} \leqslant \mathrm{O}\left(n^{2} \mathrm{e}^{-\gamma(h / 2)^{3} n}\right) .
\end{aligned}
$$

Therefore, $P\left[\exists X_{i_{1}} X_{i_{2}}\right.$ such that $d\left(X_{i_{1}}, X_{i_{2}}\right) \geqslant h$ and $\left.X_{i_{1}} X_{i_{2}} \in \mathcal{D}\right] \leqslant \mathrm{O}\left(n^{4} \mathrm{e}^{-\gamma(h / 2)^{3} n}\right)$. This is o $\left(1 / n^{4}\right)$ if

$$
h>\left(\frac{32}{\gamma} \frac{\log n}{n}\right)^{1 / 3} \text {. }
$$

Proof of Lemma 6. By Euler's formula, there is a constant $K$ such that the tetrahedron-degree is equal to $K$ times the edge-degree. For convenience, assume that $B_{2}$ denotes the event $\left\{\forall i, d^{o}\left(X_{i}\right) \leqslant\right.$ $\left.c^{\prime \prime} \log n / \log \log n\right\}$, which is identical to the event $\left\{\forall i\right.$, tetrahedron- $\left.d^{o}\left(X_{i}\right) \leqslant K c^{\prime \prime} \log n / \log \log n\right\}$. Then, from Lemma 7 it follows that $P\left[\overline{B_{2}}\right] \leqslant 1 / n^{3}$ and we have $E\left[N_{2}\right]=E\left[N_{2} ; B_{2}\right]+E\left[N_{2} ; \overline{B_{2}}\right]$. The two terms $E\left[N_{2} ; B_{2}\right]$ and $E\left[N_{2} ; \overline{B_{2}}\right]$ need to be bounded separately. We begin with $E\left[N_{2} ; B_{2}\right]$ :

\footnotetext{
${ }^{6}$ Theorem 1 of [3] establishes that the maximum edge length is at most $\mathrm{O}\left((\log n)^{1 / 3}\right)$ with high probability. Our additional factor $1 / n^{1 / 3}$ comes from the fact that they consider a cube of variable side length $1 / n^{1 / 3}$.
} 


$$
\begin{aligned}
E\left[N_{2} ; B_{2}\right] & =P\left[B_{2}\right], E\left[N_{2} \mid B_{2}\right] \\
& \leqslant K c^{\prime \prime} P\left[B_{2}\right] E\left[N_{1} \mid B_{2}\right] \log n / \log \log n \\
& =K c^{\prime \prime} E\left[N_{1} ; B_{2}\right] \log n / \log \log n \\
& \leqslant K c^{\prime \prime} E\left[N_{1}\right] \log n / \log \log n \\
& \leqslant K c^{\prime \prime}\left(b+c r^{2} n^{2 / 3}+d n r^{3}\right) \log n / \log \log n .
\end{aligned}
$$

On the other hand,

$$
E\left[N_{2} ; \overline{B_{2}}\right] \leqslant \mathrm{O}\left(n^{2}\right) P\left[\overline{B_{2}}\right] \leqslant \mathrm{O}\left(n^{2}\right) \frac{1}{n^{3}}=\mathrm{o}(1) .
$$

Corollary 8. Consider $n$ points $X_{1}, \ldots, X_{n}$ drawn independently from an $(\alpha, \beta)$-measure over a bounded convex set $C$. Then there exist positive constants $a, b, c, d$ and $e$, depending upon $\alpha, \beta$ and $C$ only, such that the following holds. Let $y$ be any point in $C$ at distance $a(\log n / n)^{1 / 3}$ from the boundary $\partial C$. Let $r, r \leqslant a /\left(2 n^{1 / 3}\right)$ be a positive quantity. Let $N_{2}$ denote the number of Delaunay tetrahedra that intersect $\mathcal{B}(y, r)$. Then

$$
E\left[N_{2}\right] \leqslant e\left(b+c(a / 2)^{2}+d(a / 2)^{3}\right) \log n / \log \log n .
$$

\subsection{Summing it all up}

We are ready to prove the theorems.

Proof of Theorem 2. We set $c_{1}=a$, where $a$ is the constant of Lemma 5 and Corollary 8. The segment $L$ may be covered by $\left\lceil(|L| / a) n^{1 / 3}\right\rceil$ circles of radius $a /\left(2 n^{1 / 3}\right)$ each and centered on points $y_{i}$ of $L$. The number $N$ of intersections between $L$ and the Delaunay triangulation is bounded by the sum of the number of intersections with these circles. By Corollary 8 , the expected number of intersections with each of these circles is bounded by $K \log n / \log \log n$ for some constant $K$ depending upon $\alpha, \beta$ and $C$ only. Hence,

$$
E[N] \leqslant K\left\lceil\frac{|L|}{a} n^{1 / 3}\right\rceil \log n / \log \log n \leqslant K\left(1+\frac{|L|}{a} n^{1 / 3}\right) \log n / \log \log n,
$$

which proves Theorem 2 .

Proof of Theorem 1. We have in mind to apply Theorem 2 to the segment $L=q Y$. We are faced with two difficulties. First, both $Y$ and $L$ are defined in terms of $Y_{1}, \ldots, Y_{m}$ and are therefore not independent of $X_{1}, \ldots, X_{n}$. Second, $Y$ can possibly be within distance $c_{4}(\log n / n)^{1 / 3}$ from the boundary $\partial C$. We will solve the first difficulty by considering a slightly different Delaunay triangulation with respect to which $L$ is independent. We will solve the second difficulty by showing that $Y$ is with high probability at distance of at least $c_{4}(\log n / n)^{1 / 3}$ from $\partial C$.

Let us first recall that $q$ and $Y$ are defined in very different ways. The condition that they be "far enough" from the boundary must therefore be handled differently. The query point $q$ is not in the control of the algorithm. It is instead decided externally and the algorithm is claimed to perform well for all admissible choices of $q$. Thus, the assumption " $q$ is at distance of at least $c_{1} / n^{1 / 18}$ from $\partial C$ " is merely a restriction on the set of query points against which the algorithm has to measure. On the other hand, the point $Y$ is chosen randomly, as described in the algorithm in Section 1 . The fact that " $Y$ is at distance of at least $c_{4}(\log n / n)^{1 / 3}$ from $\partial C$ " cannot therefore be imposed externally. 
Let us relabel $\left\{X_{1}, \ldots, X_{n}\right\}-\left\{Y_{1}, \ldots, Y_{m}\right\}$ into $\left\{X_{1}^{\prime}, \ldots, X_{n-m}^{\prime}\right\}$. As usual, let $\mathcal{D}$ denote the Delaunay triangulation associated to the $n$ points $X_{1}, \ldots, X_{n}$, and let $\mathcal{D}^{\prime}$ denote the Delaunay triangulation associated to the $n-m$ points $X_{1}^{\prime}, \ldots, X_{n-m}^{\prime}$. The random variables $X_{1}^{\prime}, \ldots, X_{n-m}^{\prime}$ are independent from the random variables $Y_{1}, \ldots, Y_{m}$. This implies that, for every query point $q,\left(X_{1}^{\prime}, \ldots, X_{n-m}^{\prime}\right)$ is independent from $Y$, which allows us to make the following two conclusions. First, $L \stackrel{\text { def }}{=}(Y, q)$, the line segment connecting $Y$ and $q$, is independent of the $n-m$ data points defining $\mathcal{D}^{\prime}$. Second, the probabilistic behavior of $X_{1}^{\prime}, \ldots, X_{n-m}^{\prime}$ is unaffected when conditioning on the event $B_{3} \stackrel{\text { def }}{=}\left\{d(Y, \partial C) \geqslant c_{4}(\log n / n)^{1 / 3}\right\}$. In formal terms, the probabilistic law $\mathcal{L}\left(X_{1}^{\prime}, \ldots, X_{n-m}^{\prime}\right)$ is equal to the conditional law $\mathcal{L}\left(\left(X_{1}^{\prime}, \ldots, X_{n-m}^{\prime}\right) \mid B_{3}\right)$. In particular, the random variables $X_{1}^{\prime}, \ldots, X_{n-m}^{\prime}$ are independent identically distributed $(\alpha, \beta)$-random variables under the conditional probability distribution $P\left[\cdot \mid B_{3}\right]$.

Let $N$ denote the number of tetrahedra in $\mathcal{D}^{\prime}$ crossed by $L$. We have $E[N]=E\left[N ; B_{3}\right]+E\left[N ; \overline{B_{3}}\right]$ where $\overline{B_{3}}$ denotes the complement of $B_{3}$. We provide upper bounds for the two terms $E\left[N ; B_{3}\right]$ and $E\left[N ; \overline{B_{3}}\right]$.

We begin with $E\left[N ; \overline{B_{3}}\right]$. It is well known that $N=\mathrm{O}\left(n^{2}\right)$. Hence $E\left[N ; \overline{B_{3}}\right] \leqslant \mathrm{O}\left(n^{2}\right) P\left[\overline{B_{3}}\right]$. We fix $c$ to be a constant such that, for every $n, c_{4}(\log n / n)^{1 / 3} \leqslant(c / 2)(1 / n)^{1 / 18}$. Recall that $P\left[\overline{B_{3}}\right]=P[d(Y, \partial C)<$ $c_{4}(\log n / n)^{1 / 3}$ ] and that, by assumption, $d(q, \partial C) \geqslant c(1 / n)^{1 / 18}$. By triangular inequality, this implies that

$$
\begin{aligned}
P\left[\overline{B_{3}}\right] & \leqslant P\left[d(Y, q) \geqslant \frac{c}{2} \frac{1}{n^{1 / 18}}\right]=\left(P\left[d\left(Y_{1}, q\right) \geqslant \frac{c}{2} \frac{1}{n^{1 / 18}}\right]\right)^{m} \\
& =\left(1-P\left[d\left(Y_{1}, q\right) \leqslant \frac{c}{2} \frac{1}{n^{1 / 18}}\right]\right)^{m} \leqslant \mathrm{e}^{-m P\left[d\left(Y_{1}, q\right) \leqslant(c / 2)\left(1 / n^{1 / 18}\right)\right]} \\
& \leqslant \mathrm{e}^{-n^{1 / 5} \alpha(4 \pi / 3)(c / 2)^{3}\left(1 / n^{1 / 6}\right)}=\mathrm{o}\left(1 / n^{2}\right) .
\end{aligned}
$$

This shows that $E\left[N ; \overline{B_{3}}\right]=\mathrm{o}(1)$. We now turn to $E\left[N ; B_{3}\right]=E\left[N \mid B_{3}\right] P\left[B_{3}\right]$. Theorem 2 (see the remark after Theorem 2), along with the fact that $X_{1}^{\prime}, \ldots, X_{n-m}^{\prime}$ are $(\alpha, \beta)$-random variables, independently identically distributed under the measure $P\left[\cdot \mid B_{3}\right]$, implies that

$$
E\left[N \mid B_{3}\right] \leqslant c_{4}+c_{5} E\left[d(Y, q) \mid B_{3}\right](n-m)^{1 / 3} \log n / \log \log n .
$$

Hence,

$$
\begin{aligned}
E\left[N ; B_{3}\right] & \leqslant c_{4} P\left[B_{3}\right]+c_{5} E\left[d(Y, q) ; B_{3}\right](n-m)^{1 / 3} \log n / \log \log n \\
& \leqslant c_{4}+c_{5} E[d(Y, q)] n^{1 / 3} \log n / \log \log n .
\end{aligned}
$$

The estimation of $E[d(Y, q)]$ is done as in [10]. The beginning of the argument is similar to the estimation of $P\left[\overline{B_{3}}\right]$ above. Lemma 4 is then used. We let diam $(C)$ denote the diameter of $C$. Note that $Y$ and $q$ are in $C$ so that $P[d(Y, q)>t]=0$ if $t>\operatorname{diam}(C)$.

$$
\begin{aligned}
E[d(Y, q)] & =\int_{0}^{\infty} P[d(Y, q)>t] \mathrm{d} t \leqslant \int_{0}^{\infty} \mathrm{e}^{-m P\left[d\left(Y_{1}, q\right) \leqslant t\right]} \mathrm{d} t \leqslant \int_{0}^{r_{0}} \mathrm{e}^{-m \alpha \gamma t^{3}} \mathrm{~d} t+\int_{r_{0}}^{\operatorname{diam}(C)} \mathrm{e}^{-m \alpha \gamma r_{0}{ }^{3}} \mathrm{~d} t \\
& \leqslant \int_{0}^{\infty} \mathrm{e}^{-m \alpha \gamma t^{3}} \mathrm{~d} t+\operatorname{diam}(C) \mathrm{e}^{-m \alpha \gamma r_{0}^{3}}=\mathrm{O}\left(\frac{1}{m^{1 / 3}}\right) .
\end{aligned}
$$


We have therefore shown that $E[N]=\mathrm{O}\left((n / m)^{1 / 3} \log n / \log \log n\right)$.

$N_{\text {total }}$, the total number of tetrahedra in $\mathcal{D}$ crossed by $L$, is not more than that for $\mathcal{D}^{\prime}$, that is, above $N$, plus the sum $S$ of the tetrahedra degrees of (that is, the number of tetrahedra adjacent to) $Y_{1}, \ldots, Y_{m}$ in the Delaunay triangulation $\mathcal{D}$. To see this, note that $L$ either crosses a tetrahedron without one of the $Y_{i}$ 's as a vertex (in which case the tetrahedron is both in $\mathcal{D}$ and $\mathcal{D}^{\prime}$ ) or one for which $Y_{i}$ is a vertex (in which case the tetrahedron is in $\mathcal{D}$ but not in $\mathcal{D}^{\prime}$ ). The total number of the latter kind of tetrahedra does not exceed $S$. The expected value of $S$ is, by linearity of expectation, $3 m$ times the expected (vertex) degree $\delta(n)$ of $Y_{1}$, where the constant 3 results from Euler's formula. Combining all this we have

$$
E\left[N_{\text {total }}\right]=\mathrm{O}\left((n / m)^{1 / 3} \log n / \log \log n+m \delta(n)\right) .
$$

The time complexity $T$ of the jump-and-march algorithm in Section 1 is proportional to $m+N_{\text {total }}$; the sample size $m$ comes into play because of steps (1) and (2), $N_{\text {total }}$ is due to step (3). E[T] can thus be optimized to $\mathrm{O}\left(\delta(n)^{1 / 4} n^{1 / 4}(\log n / \log \log n)^{3 / 4}\right)$ with the choice of $m=\Theta\left(n^{1 / 4} / \delta(n)^{3 / 4}(\log n /\right.$ $\log \log n)^{3 / 4}$ ).

\section{Empirical results in $2 D$}

This section presents some empirical results on the planar jump-and-march, or better, a variation of it. For further convenience, we sample $n^{1 / 3}$ edges of the Delaunay triangulation $\mathcal{D}$, rather than points. Then, we choose the edge whose midpoint has minimum distance to the query point $q$. We find the triangle containing $q$ by traversing the triangles intersected by $L=(y, q)$, where $y$ is the midpoint of the initially chosen edge.

We tested this procedure for random point sets of size $n=1000,2000, \ldots, 50000$; the coordinates were chosen randomly out of the unit square. In Figs. 8(a) and (b), $M_{n}$ denotes the sample mean of the number of triangles visited, over a sample of 999 queries, and for one random point set of size $n$, for each $n$; the coordinates of $q$ (and the point set) are chosen by random out of the unit square. Thus, $M_{n}$ corresponds to the $E\left[N_{\text {total }}\right]$ in the analysis. Since $\Theta(\log n)$ is the best known theoretical bound for planar point locations; see, for example, [21], Fig. 8(c) plots the ratio $M_{n} / \log _{2} n$ to give a measure for

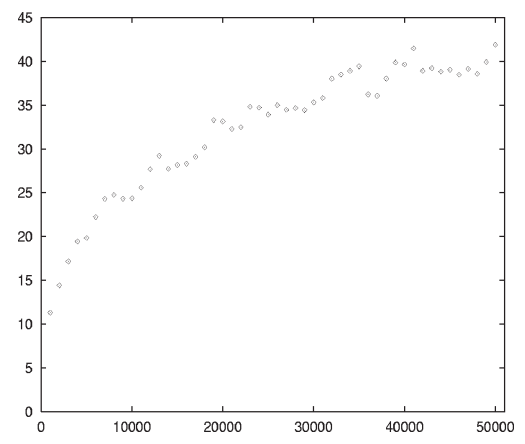

(a)

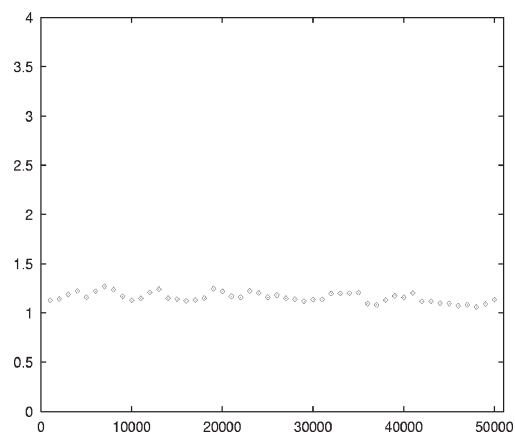

(b)

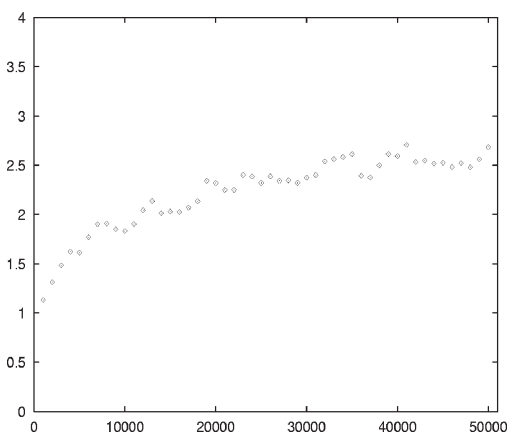

(c)

Fig. 8. (a) The sample mean $M_{n}$ of the number of triangles visited, for a sample of 999 random query points $q$ for each data set of size $n$. (b) The ratio $M_{n} / n^{1 / 3}$. (c) The ratio $M_{n} / \log _{2} n$. 
the efficiency of the method. Note that the best known planar point location algorithm [12] is obtained by two binary searches, one horizontally and one vertically, thus has at least a constant of 2 in front of the $\log _{2} n$.

\section{Empirical results in 3D}

How does the jump-and-march perform in 3D? Again, we implement a slight variation of the procedure analyzed in this text; the empirical studies listed here, but also in the context of incremental Delaunay triangulators [20], justify this. In order to discuss the procedure, it helps to have the concept of an oriented triangle $\tau$. In essence, it is given by the (ordered) sequence of its vertices. The vertices then define (the underlying plane with) a normal vector pointing, by definition, to the triangle's positive side, denoted by $\tau^{+}$. Linear algebra dictates that a triangle has two distinguishable orientations.

With this, our variant, the jump-and-walk, works as follows. First, we sample triangles rather than vertices. The sample size is set to $m=\mathrm{O}\left(n^{1 / 4}\right)$, for Delaunay triangulations of $n$ points. The "distance" of a triangle to the query point $q$ is calculated as the minimum distance of its three vertices to $q$. The triangle $\tau_{0}$ which scores with the shortest distance is selected. We adjust its orientation such that $q$ is on its positive side, that is, $q \in \tau_{0}^{+}$. The selection of $\tau_{0}$ constitutes the "jump" part of the algorithm.

Second, we perform the following loop, which implements the original "walking" strategy mentioned in the introduction. The loop has the invariant that $q \in \tau^{+}$.

(1) If $\tau$ is a convex hull triangle and $\tau^{+}$is outside $\mathcal{D}$, then $q$ lies outside $\mathcal{D}$.

Exit loop.

(2) Otherwise, there is a tetrahedron in $\mathcal{D}$ incident to $\tau$ (and in $\tau^{+}$).

If this tetrahedron contains $q$, exit loop.

(3) Otherwise, select a triangle $\sigma$ of the tetrahedron, such that, $q \in \sigma^{+}$.

(4) Set $\tau=\sigma$, and continue loop at (1).

Each iteration of this loop corresponds to a tetrahedron visited. Again, note that this procedure is only guaranteed to terminate for Delaunay triangulations since they are proven to be "acyclic for any fixed viewpoint" [14]. For arbitrary triangulations, this is not necessarily the case; however, if the selection of $\sigma$ in (3) is done by random (out of the up to 3 possibilities), then the infinite loop is broken with probability arbitrary close to 1 .

In terms of the number of tetrahedra visited, jump-and-walk can obviously be only worse than jumpand-march. However, orientation tests in 3D are computationally less involved than intersection tests; and this is particular true, if the implementation emphasizes robustness. Therefore, it is not surprising that experiments reveal the jump-and-walk to actually run faster in practice.

For our experiments, we generated 5 random 3D point sets for each $n=1000,2000, \ldots, 50000$. Each data set was then queried with 9999 random points, using the jump-and-walk algorithm. We chose $m=7 n^{1 / 4}$ for the random sample. This was empirically determined to be the best choice with respect to actual CPU time. It is obvious that the larger $m$, the smaller the number of tetrahedra visited in the walk. For $m=2 n^{1 / 4}$, these numbers roughly balance. However, it makes sense to increase $m$, because the sampling of a triangle in the jump phase is computationally less expensive than the visit of a tetrahedron while walking towards the query point. This is so in particular because care has to be taken to implement the walk robustly, for example, using symbolic perturbation and exact arithmetic. 

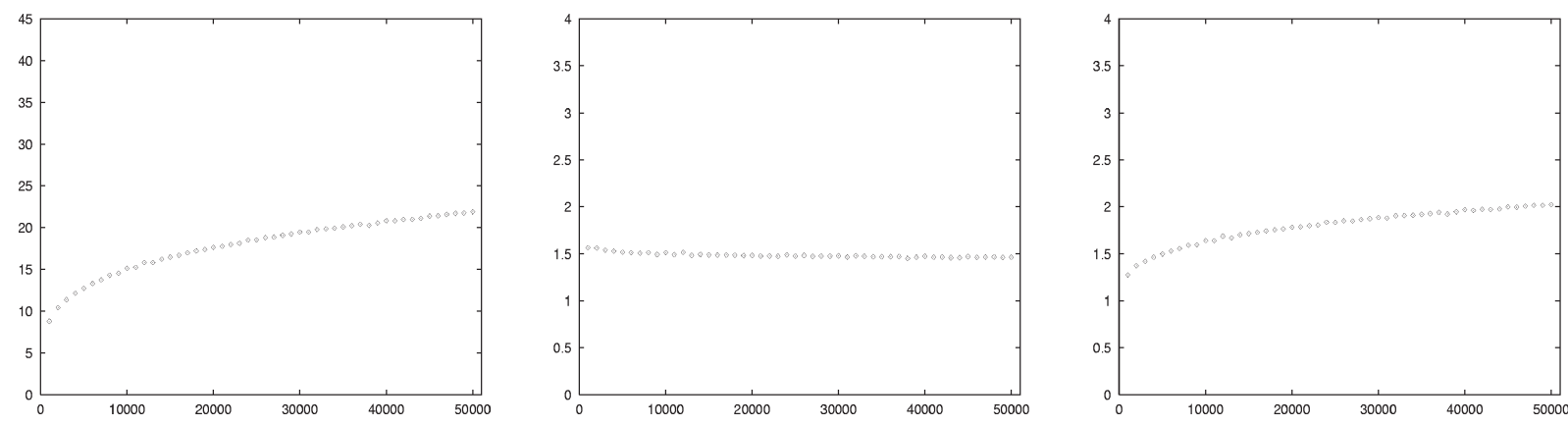

Fig. 9. (a) The sample mean $M_{n}$ of the number of tetrahedra visited, for a sample of 9999 random query points $q$ for a random point set of size $n$. The plotted value $M_{n}$ reflects the worst mean out of 5 random point sets for each $n$. (b) The ratio $M_{n} / n^{1 / 4}$. (c) The ratio $M_{n} / \log _{2} n$.

For each of the 9999 query points we count the number of tetrahedra visited and take the mean. This gives us 5 sample mean values for each $n$; Fig. 9(a) plots $M_{n}$, that is, the largest of the 5 . The corresponding $90 \%$ confidence intervals were consistently smaller than $\pm 1 \%$. Here, we say that the sample mean $\hat{\mu}$ has a $90 \%$ confidence interval of $\pm H \%$ if the interval $[\hat{\mu}(1-H / 100), \hat{\mu}(1+H / 100)]$ contains the real mean $\mu$ with probability at least $90 \%$.

Fig. 9(b) plots the ratio $M_{n} / n^{1 / 4}$. It indicates that the constants in our analysis are low, that is, less than 1.6. Moreover, the method compares well with the theoretically best possible $\mathrm{O}(\log n)$, which assumes both preprocessing and additional storage. Fig. 9(c) plots $M_{n} / \log _{2} n$ and shows that, for the observed range of $n$, the number of visited tetrahedra stays well under $2.1 \log _{2} n$.

\section{Closing remarks}

Point location by walking through a triangulation is used in the practice of geometric computing for years, and with excellent empirical results; in particular in 2D or 3D mesh generation; see, for example, [22] or [5,16,24], respectively. More recently, following the original conference presentation of this work, marching or walking through triangulations or subdivisions is finally also finding the attention of the more theoretical computational geometry community; see, for example, [1,9,17].

In this paper, following the strict requirement of no additional storage, and by simulating bucketing via random sampling, we enhanced the procedure to what we call the jump-and-march. We were able to show that the new procedure has an expected running time of $\mathrm{O}\left(n^{1 / 4}\right)$ when applied to the 3D Delaunay triangulation of a uniformly distributed random point set of size $n$. Our own empirical tests verify this; in fact, the relaxed jump-and-walk procedure, which is even easier to implement, performs as good, or even better in terms of actual running time. Experiments with nonrandom data show similar results [20]. Our work justifies a procedure which is often used in the "real world" and even suggests a simple yet effective improvement.

Our work raises several interesting questions both theoretical and empirical.

(1) Our result shows that the method requires expected time close to $\mathrm{O}\left(n^{1 /(d+1)}\right)$ for $d=3$ dimensions. It would be very interesting to settle whether the method performs equally well for higher dimensions. Two things are required to adapt our proof to higher dimensions. First, in order to extend our 
Lemma 5 and bound the expected value of $N_{1}$, one needs to define appropriately a " $d$-dimensional spindle": this geometrical object is composed of $d$ balls whose centers are on a $d-1$-sphere positioned on the hyperplane between $X_{1}$ and $y$. A critical step is to provide a $d$-dimensional version of our Lemma 3. Second, one needs to provide a $d$-dimensional replacement to the Euler argument that allowed us to relate $N_{2}$ to $N_{1}$. A complete different approach might be possible. Indeed, we believe that our results can be tightened and that

(2) in all our results the $\log n / \log \log n$ terms can be removed.

(3) Our results make explicit the perturbing influence of the boundary. The proof of Lemma 5 required to identify locally the boundary $\partial C$ to a plane. It would be very interesting to quantify this in terms of the curvature of $\partial C$.

(4) It would be extremely interesting to know how the method performs on non-Delaunay triangulations.

(5) Finally, it would be very interesting to construct an adaptive version of our algorithm in the context where many query points are considered. One possibility would be to incrementally improve the data-structure as more and more query points are located. This would allow to select the $m$ points of phase (1) in a more optimal way than purely random, resulting in a point $Y$ closer to the query point. The improved algorithm could be analyzed via amortized analysis.

\section{References}

[1] B. Aronov, S. Fortune, Average-case ray shooting and minimum weight triangulations, in: Proceedings of the 13th Annual Symposium on Computational Geometry, 1997, pp. 203-212.

[2] T. Asano, M. Edahiro, H. Imai, M. Iri, K. Murota, Practical use of bucketing techniques in computational geometry, in: G.T. Toussaint (Ed.), Computational Geometry, North-Holland, Amsterdam, 1985, pp. 153195.

[3] M. Bern, D. Eppstein, F. Yao, The expected extremes in a Delaunay triangulation, Internat. J. Comput. Geom. Appl. 1 (1991) 79-91.

[4] J. Bonet, J. Peraire, An alternating digital tree (ADT) algorithm for 3D geometric searching and intersection problems, Internat. J. Numer. Methods Engrg. 31 (1991) 1-17.

[5] H. Borouchaki, S.H. Lo, Fast Delaunay triangulation in three dimensions, Comput. Methods Appl. Mech. Engrg. 128 (1-2) (1995) 153-167.

[6] P. Bose, L. Devroye, Intersections with random geometric objects, Manuscript, School of Computer Science, McGill University, 1995.

[7] A. Bowyer, Computing Dirichlet tessellations, The Computer Journal 24 (1981) 162-166.

[8] E. Brisson, Representing geometric structures in $d$ dimensions: Topology and order, Discrete Comput. Geom. 9 (4) (1993) 387-426.

[9] O. Devillers, Improved incremental randomized Delaunay triangulation, Technical Report 3298, INRIA, Sophia-Antipolis, 1997.

[10] L. Devroye, E. Mücke, B. Zhu, A note on point location in Delaunay triangulations of random points, Algorithmica (Special Issue on Average Case Analysis of Algorithms) 22 (4) (1998) 477-482.

[11] D.P. Dobkin, M.J. Laszlo, Primitives for the manipulation of three-dimensional subdivisions, Algorithmica 4 (1) (1989) 3-32.

[12] D.P. Dobkin, R.J. Lipton, Multidimensional searching problems, SIAM J. Comput. 5 (1976) 181-186.

[13] R.A. Dwyer, Higher-dimensional Voronoi diagrams in linear expected time, Discrete Comput. Geom. 6 (1991) 343-367.

[14] H. Edelsbrunner, An acyclicity theorem for cell complexes in $d$ dimensions, Combinatorica 10 (1990) 251260. 
[15] M. Garland, P.S. Heckbert, Fast polygonal approximation of terrains and height fields, Technical Report CMUCS-95-181, School of Computer Science, Carnegie Mellon University, 1995.

[16] P.L. George, F. Hermeline, Delaunay's mesh of a convex polyhedron in dimension $d$, Application to arbitrary polyhedra, Internat. J. Numer. Methods Engrg. 33 (5) (1992) 975-995.

[17] M.T. Goodrich, M. Orletsky, K. Ramaiyer, Methods for achieving fast query times in point location data structures, in: Proceedings of 8th Annual ACM-SIAM Symposium on Discrete Algorithms (SODA '97), 1997, pp. 757-766.

[18] P.J. Green, R. Sibson, Computing Dirichlet tessellations in the plane, The Computer Journal 21 (1978) 168173.

[19] L.J. Guibas, J. Stolfi, Primitives for the manipulation of general subdivisions and the computation of Voronoi diagrams, Trans. Graphics 4 (2) (1985) 74-123.

[20] E.P. Mücke, Shapes and implementations in three-dimensional geometry, Ph.D. Thesis, Technical Report UIUCDCS-R-93-1836, Department of Computer Science, University of Illinois at Urbana-Champaign, Urbana, IL, 1993.

[21] F.P. Preparata, M.I. Shamos, Computational Geometry: An Introduction, Springer, Berlin, 1985.

[22] J.R. Shewchuk, Triangle: Engineering a 2D quality mesh generator and Delaunay triangulator, in: Proceedings of the 1st ACM Workshop on Applied Computational Geometry, 1996, pp. 124-133.

[23] J. Snoeyink, Point location, in: J.E. Goodman and J. O'Rourke (Eds.), Handbook of Discrete and Computational Geometry, CRC Press, Boca Raton, FL, 1997, pp. 559-574.

[24] H. Trease, D. George, C. Gable, J. Fowler, E. Linnbur, A. Kuprat, A. Khamayseh, The X3D Grid Generation System, in: Proceedings of the 5th International Conference on Numerical Grid Generation in Computational Field Simulations, 1996, pp. 239-244. 